\title{
Scribble complex protein deficiency promotes prostate tumors in vivo
}

The Scribble complex is one of several protein complexes involved in maintaining cellular polarity. As loss of polarity is characteristic of epithelial cancers, polarity regulators could be important in the prevention of tumorigenesis. An Australian team have now investigated this hypothesis in prostate cancer in vivo, publishing their data in the Journal of Clinical Investigation.

Cellular polarity is maintained by regulators such as the interacting complexes Scribble, Crumbs and Par, which mediate migration, asymmetric cell division and planar cell modality. In vitro data suggest that deregulation of Scribble complex proteins is associated with human epithelial cancers-an effect thought to be directly mediated by oncogenic pathways such as the Ras-MAPK cascade. Thus far, evidence of the effect of Scrib as a regulator of tumorigenesis in vivo has been lacking.

The team first generated Scrib knockout mice, finding that global Scrib knockout resulted in perinatal death owing to incomplete neural tube closure. These mice also had curly tails and gonadal abnormalities as well as gastroschisis. 70\% of mice heterozygous for Scrib displayed prostate hyperplasia, supporting the hypothesis that loss of polarity can result in tumorigenesis. These effects were associated with an increase in Ras-MAPK signaling, suggesting that SCRIB deficiency upregulates this cascade.

Studies using mice with conditional prostatic Scrib knockout-that is, Scrib knocked out solely in the prostate epithelium-had multifocal prostatic hyperplasia. Biallellic Scrib loss also facilitated neoplastic progression,

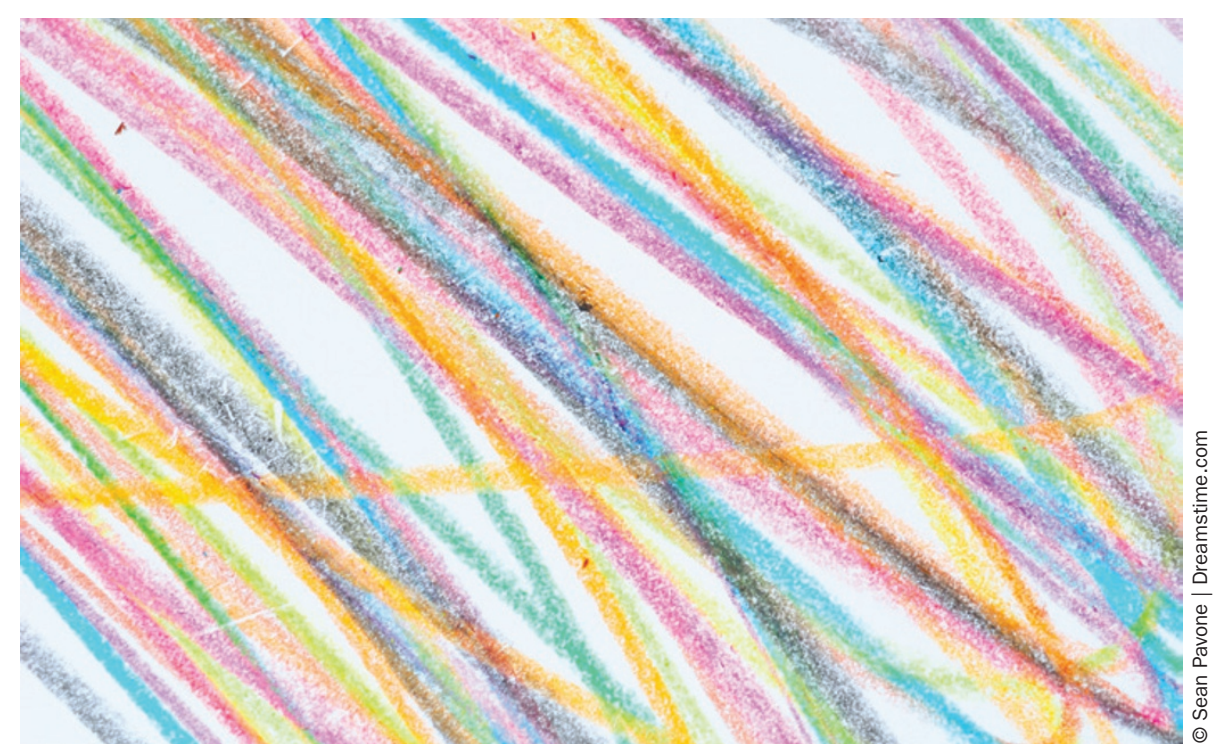

with areas of prostatic intraluminal neoplasia formed in association with the hyperplastic regions. Once again, the RasMAPK cascade was upregulated, further supporting the hypothesis that Scrib deregulation leads to prostatic neoplasia via this pathway. Furthermore, the researchers also showed that Scrib loss and activation of oncogenic Ras cooperated to drive progression of prostate tumors-an effect previously shown in Drosophila.

Finally the authors investigated SCRIB expression in samples of human prostate cancer tissue. Using tissue microarrays and immunohistochemistry, they tested samples of over 2,000 patients with hormone-naïve prostate cancer. SCRIB signal intensity was correlated with tumor stage, grade and patient PSA level.

"The observation that even subtle changes in cell polarity can drive disorganization and prostate hyperplasia is a powerful demonstration of how sensitive glandular tissue can be to insults in tissue organization," comments Patrick Humbert, who led the study. Demonstrating the link between polarity and cancer, these data raise interesting questions regarding mechanisms of prostate tumorigenesis and whether polarity regulators could one day provide a useful therapeutic target for prostate cancer.

Annette Fenner

Original article Pearson, H. B. et al. SCRIB expression is deregulated in human prostate cancer and its deficiency in mice promotes prostate neoplasia. J. Clin. Invest. doi:10.1172/JCI58509 